\title{
Evolution of newborn rapidly rotating magnetars: effects of $R$-mode and fall-back accretion
}

\author{
Jie-Shuang Wang ${ }^{1,2,3}$ and Zi-Gao Dai ${ }^{1,2 \star}$ \\ ${ }^{1}$ School of Astronomy and Space Science, Nanjing University, Nanjing 210093, China \\ 2 Key Laboratory of Modern Astronomy and Astrophysics (Nanjing University), Ministry of Education, Nanjing 210093, China \\ ${ }^{3}$ Max-Planck-Institut für Kernphysik, Saupfercheckweg 1, D-69117 Heidelberg, Germany
}

Preprint online version: November 13, 2021

\section{ABSTRACT}

\begin{abstract}
In this paper we investigate effects of the $r$-mode instability on a newborn rapidly-rotating magnetar with fall-back accretion. Such a magnetar could usually occur in core-collapse supernovae and gamma-ray bursts. We find that the magnetar's spin and $r$-mode evolution are influenced by accretion. If the magnetar is sufficiently spun up to a few milliseconds, gravitational radiation leads to the growth of the $r$-mode amplitude significantly. The maximum $r$-mode amplitude reaches an order $\sim 0.001$ when the damping due to the growth of a toroidal magnetic field balances the growth of the $r$-mode amplitude. If such a sufficiently spun-up magnetar was located at a distance less than $1 \mathrm{Mpc}$, then gravitational waves would be detectable by the Einstein Telescope but would have an extremely low event rate. However, if the spin-up is insufficient, the growth of the $r$-mode amplitude is mainly due to the accretion torque. In this case, the maximum $r$-mode amplitude is of the order of $\sim 10^{-6}-10^{-5}$.
\end{abstract}

Key words. gravitational waves - stars: neutron - stars: rotation - stars: oscillations - stars: magnetars - accretion, accretion discs

\section{Introduction}

The $r$-mode instability is of great astrophysical importance, since it occurs in all rotating perfect fluid neutron stars (Andersson 1998; Friedman \& Morsink 1998). This instability is driven by gravitational radiation via the Chandrasekhar-Friedman-Schutz mechanism (Chandrasekhar 1970; Friedman \& Schutz 1978), which leads to rapid growth of the $r$-mode amplitude, while viscosity acts to suppress the instability. Given the dependence of gravitational wave emission on the neutron star spin and the dependence of viscosity on the neutron star temperature, the critical angular velocity-temperature curve dividing the $r$-mode stability from instability (hereafter $r$-mode instability window) was obtained by Lindblom et al. (1998), who also found that gravitational radiation of the $l=2$ current multipole is stronger than the other multipoles. Owen et al. (1998) carried out a detailed study of the $r$-mode instability in rapidly rotating neutron stars and calculated the gravitational wave forms. Such gravitational wave signals might be detected at a distance of $20 \mathrm{Mpc}$ by the advanced Laser Interferometer Gravitational-wave Observatory (aLIGO). Further studies were carried out to obtain the properties of the $r$ mode instability especially for gravitational waves from rotating neutron stars (Andersson et al. 1999; Kokkotas \& Stergioulas 1999; Lindblom et al. 1999; Andersson et al. 2000; Bildsten \& Ushomirsky 2000; Lindblom \& Mendell 2000; Lindblom et al. 2000, 2001; Andersson et al. 2001; Arras et al. 2003; Bondarescu et al. 2007, 2009; Yang et al. 2010; Haskell et al. 2012; Alford \& Schwenzer 2014a b, 2015; Chugunov 2015; Haskell 2015; Mytidis et al. 2015) and rotating strange stars (Madsen 1998, 2000; Andersson et al. 2002; Zheng et al. 2006; Alford \& Schwenzen 2014b; Dai et al. 2016).

\footnotetext{
^ E-mail:dzg@nju.edu.cn
}

Neutron stars form during supernova (SN) explosions. Fallback accretion could happen after the core collapse of massive stars (Michel 1988; Chevalier 1989). Owing to rotating progenitors, a disk is likely to form before fall-back materials are accreted onto the neutron star surface (e.g., we refer to Piro \& Ott 2011). Such a fall-back disk is responsible for the jets of pulsars (Blackman \& Perna 2004), and early afterglows of gamma-ray bursts (GRBs; Dai \& Liu 2012). The $r$ mode instability of neutron stars (with sub-Eddington accretion) in low-mass X-ray binary (LMXB) systems was investigated (Levin 1999; Andersson et al. 1999; Brown \& Ushomirsky 2000; Haskell et al. 2012; Mahmoodifar \& Strohmaver 2013). The accreted materials in LMXBs spin up old neutron stars significantly and cause these stars to enter the $r$-mode instability window again. Following this, the heating due to shear viscosity affects the stellar thermal evolution while gravitational radiation spins down the star. The effects of hypercritical advectiondominated accretion flows without magnetic fields in close binary systems were investigated by Yoshida \& Eriguchi (2000), who found that hyperaccreting disks considerably increase the stellar angular momentum, which can balance the spin-down due to gravitational radiation.

Newborn neutron stars are generally strongly magnetized. The average magnetic field of isolated radio pulsars is $\sim 2 \times 10^{12}$ $\mathrm{G}$ (Arzoumanian et al. 2002). However, some astrophysical phenomena were driven by magnetars with field strengths up to $10^{16} \mathrm{G}$, for example GRBs (Usov 1992; Dai \& Lu 1998a b; Zhang \& Mészáros 2001; Thompson et al. 2004; Metzger et al. 2007; Dall'Osso et al. 2011), soft gamma-ray repeaters (SGRs, Thompson \& Duncan 1995, 1996; Vasisht \& Gotthelf 1997; Kouveliotou et al. 1998) and some superluminous supernovae (Kasen \& Bildsten 2010; Woosley 2010; Wang et al. 2015). Based on the Australia Telescope National Facility (ATNF) cat- 
alog (Manchester et al. 2005), the highest magnetic field discovered so far is $\sim 2 \times 10^{15} \mathrm{G}$ in SGR 1806-20. Such an ultrastrong magnetic field plays an important role in $r$-mode evolution (Ho \& Lai 2000; Rezzolla et al. 2000, 2001a b; Lee 2005; Cuofano \& Drago 2010; Cuofano et al. 2012; Abbassi et al. 2012; Asai et al. 2015; Chugunov 2015).

Fall-back accretion of rapidly rotating magnetars could drive a subset of transients. For example, the propeller effect during fall-back accretion of newborn millisecond magnetars could directly power some broad-lined type Ib/c or IIP SNe (Piro \& Ott 2011); the early bumps of some GRB afterglows also hint that their central engines are hyper-accreting millisecond magnetars (Dai \& Liu 2012). On one hand, the $r$-mode could be unstable in these magnetars and affect the spin evolution via gravitational radiation. On the other hand, both magnetic dipole radiation and fall-back accretion can affect evolution of the stellar spin and $r$-mode amplitude. In this paper, we explore the effects of the $r$ mode instability on evolution of newborn hyper-accreting magnetars, and test whether or not gravitational waves due to the $r$ mode are detectable. If detected, such gravitational waves could provide an important probe for central engines of some $\mathrm{SNe}$ and GRBs. It was found that magnetars could be gravitational wave sources due to toroidal magnetic field-induced distortion of the neutron star (Cutler 2002; Dall'Osso et al. 2009) and the bar-mode instability (Corsi \& Mészáros 2009). Piro \& Thrane (2012) studied gravitational radiation due to bar-mode-like oscillations in accreting magnetars. They found that for a magnetar with radius $\sim 20 \mathrm{~km}$ gravitational radiation lasts between hundreds of and a few thousand seconds at typical frequencies of $1000-2000 \mathrm{~Hz}$ (Piro \& Thrane 2012). However, the $r$-mode instability could dominate gravitational radiation if the saturation amplitude is large enough. In this case, the frequencies of gravitational waves due to $r$-mode instability are $\sim 100-1000 \mathrm{~Hz}$ as is shown below, which are the most sensitive frequencies of aLIGO (Harry \& LIGO Scientific Collaboration 2010) and the Einstein Telescope (ET) (Hild et al. 2011). Thus future detections of gravitational waves could distinguish between these two kinds of oscillations.

In this paper, we investigate the effects of the $r$-mode instability on a newborn rapidly-rotating magnetar with fall-back accretion. In section 2, we present some basic equations of the $r$ mode instability and hyper-accreting disks in magnetars and then we obtain evolution equations of the stellar spin and $r$-mode amplitude. The numerical and semi-analytical results are presented in section 3 . In section 4 , we estimate the detectability of gravitational waves due to the $r$-mode instability. Our conclusions and brief discussions are given in section 5 .

\section{Basic equations of $R$-mode instability}

\subsection{Gravitational radiation and viscosities}

The $r$-mode instability is generically driven by gravitational radiation and dissipated by viscosities. The typical timescales of gravitational radiation $\left(t_{\mathrm{gw}}\right)$, shear viscosity $\left(t_{\mathrm{sv}}\right)$, and bulk viscosity $\left(t_{\mathrm{bv}}\right)$ depend on the stellar equation of state (EOS). A polytropic EOS of $N=0$ or 1 is usually used, while the resulting timescales for these two polytropic EOSs differ only by roughly a factor of two (we refer to a review by Andersson et al. (2001) and references therein). The effects of more realistic EOSs have been tested by Papazoglou \& Moustakidis (2016), whose results show only small differences from those of the $N=1$

\footnotetext{
1 http://www.atnf.csiro.au/people/pulsar/psrcat/
}

polytropic EOS. Following Alford et al. (2012a b), we consider the Akmal-Pandharipande-Ravenhall (APR) EOS (Akmal et al. 1998), since this EOS gives a better approximation of the equilibrium configuration than the polytropic EOSs for $r$-mode oscillations.

The gravitational radiation timescale is given by Lindblom et al. (1998) and Owen et al. (1998),

$$
\begin{aligned}
\frac{1}{t_{\mathrm{gw}}}= & -\frac{32 \pi G \Omega^{2 l+2}}{c^{2 l+3}} \frac{(l-1)^{2 l}}{[(2 l+1) ! !]^{2}} \\
& \times\left(\frac{l+2}{l+1}\right)^{2 l+2} \int_{0}^{R} \rho(r) r^{2 l+2} d r
\end{aligned}
$$

where $G$ is the gravitational constant. The strongest gravitational radiation reaction comes from the $l=m=2$ mode (Lindblom et al. 1998; Owen et al. 1998), so we obtain

$t_{\mathrm{gw}} \approx-24 R_{11.5}^{-4} M_{1.4}^{-1} v_{3}^{-6} \mathrm{~s}$,

where the radius of the magnetar is $R=11.5 R_{11.5} \mathrm{~km}$, the mass is $M=1.4 M_{\odot} \times M_{1.4}$, and $v=\Omega / 2 \pi=10^{3} v_{3} \mathrm{~Hz}$ is the rotational frequency of the magnetar.

Usually very simple models of shear and bulk viscosities are adopted. However, these models are inconsistent with observations of LMXBs even if taking into account the effect of an Ekman layer (Alford \& Schwenzer 2014a), and thus reevaluations of the relevant physics are needed (Ho et al. 2011; Haskell et al. 2012; Gusakov et al. 2014; Haskell 2015). Here we consider more recent and complex models to calculate the dissipation timescales of viscosities. The shear viscosity is found to be contributed by electron scattering (Shternin \& Yakovlev 2008; Shternin et al. 2013). Following Alford et al. (2012a b), we adopt this electron scattering model to calculate the dissipation timescale of shear viscosity in this paper, which is

$t_{\mathrm{sv}}=1.4 \times 10^{8} M_{1.4} R_{11.5}^{-1} T_{9}^{5 / 3} \mathrm{~s}$,

where $T=T_{9} 10^{9} \mathrm{~K}$ is the core temperature of the neutron star.

The bulk viscosity is more important, since it is the dominant dissipation mechanism for newly-born neutron stars. Most previous studies have focused on the effects of the bulk viscosity from the linear perturbation theory. However, as the $r$ mode amplitude $(\alpha)$ grows large enough $\alpha \gtrsim O(1)$, the bulk viscosity is significantly enhanced due to the suprathermal effect (Alford et al. 2012b). This effect provides an alternative mechanism to saturate the $r$-mode amplitude with the order $O(1)$ to $O(10)$ (Alford et al. 2012b). In this case, the dissipation timescale of the bulk viscosity $\left(t_{\mathrm{bv}}\right)$ also depends on the $r$-mode amplitude $\alpha$. For a neutron star dominated by the modifiedURCA processes, this time-scale is expressed as (for more details, we refer to Alford et al. 2012b),

$$
\begin{gathered}
\frac{1}{t_{\mathrm{bv}}}=1.4 \times 10^{-10} R_{11.5}^{5} M_{1.4}^{-1} T_{9}^{6} v_{3}^{2}\left(1+284.5 R_{11.5}^{4} v_{3}^{4} T_{9}^{-2} \alpha^{2}+\right. \\
\left.3.16 \times 10^{4} R_{11.5}^{8} v_{3}^{8} T_{9}^{-4} \alpha^{4}+1.08 \times 10^{6} R_{11.5}^{12} v_{3}^{12} T_{9}^{-6} \alpha^{6}\right) \mathrm{s}^{-1} .
\end{gathered}
$$

The first term on the right-hand side, namely $t_{\mathrm{bv}}^{-1}=1.4 \times$ $10^{-10} R_{11.5}^{5} M_{1.4}^{-1} T_{9}^{6} v_{3}^{2}$, is consistent with the results from the linear perturbation theory in previous studies (Lindblom et al. 1998; Owen et al. 1998).

\subsection{Effect of magnetic field}

In rotating stars, the Coriolis force $\left(F_{\mathrm{c}}\right)$ provides the restoring force. However, in strongly-magnetized neutron stars, the 
magnetic force $\left(F_{B}\right)$ also acts on the fluid motion. The effects of a magnetic field have been studied widely (Ho \& Lai 2000; Rezzolla et al. 2000, 2001a b; Lee 2005; Cuofano \& Drago 2010; Cuofano et al. 2012; Abbassi et al. 2012; Asai et al. 2015; Chugunov 2015). Differential rotation might be induced, and thus transfer energy to produce a toroidal magnetic field (Rezzolla et al. 2000, 2001a b). We consider the damping timescale due to the generation of a toroidal magnetic field following Cuofano \& Drago (2010); Cuofano et al. (2012), that is,

$t_{\mathrm{B}, \mathrm{t}} \approx 5.8 R_{11.5}^{-1} M_{1.4} B_{15}^{-1} B_{\mathrm{t}, 14}^{-1} \mathrm{v}_{3} \mathrm{~s}$,

where $B=B_{15} 10^{15} \mathrm{G}$ is the surface magnetic field of the magnetar and $B_{\mathrm{t}}=B_{\mathrm{t}, 14} 10^{14} \mathrm{G}$ is the volume-averaged toroidal magnetic field, which follows from

$\frac{d B_{\mathrm{t}}}{d t} \approx\left(\frac{4}{3 \pi}\right)^{1 / 2} B \alpha^{2} \Omega$.

A toroidal magnetic field in the interior can deform the neutron star to an ellipsoid, which leads to gravitational radiation and meanwhile spins down the star by a torque of $N_{\mathrm{B}, \mathrm{gw}}=$ $-I \Omega / t_{\mathrm{B}, \mathrm{gw}}$, where $t_{\mathrm{B}, \mathrm{gw}}$ is the timescale of gravitational radiation related to this torque, $I=\tilde{I} M R^{2}$ is the moment of inertia, and $\tilde{I}=\frac{8 \pi}{3 M R^{2}} \int_{0}^{R} \rho r^{4} d r$. It is a non-trivial problem that the gravitational radiation from such a magnetically-distorted neutron star depends on details of the magnetic field distribution in the stellar interior, as discussed by many authors (Cutler 2002; Dall'Osso et al. 2009; Corsi \& Mészáros 2009; Cuofano et al. 2012; Dall'Osso et al.2015). If the inclination angle between the magnetic dipole moment and the rotation axis is $\chi=\pi / 2$, the time-varying mass quadrupole of the neutron star and consequently the gravitational radiation efficiency are maximized; otherwise, the gravitational radiation efficiency should be smaller. If a neutron star has an initial temperature $T_{0}=10^{10} \mathrm{~K}$ and cools down via the modified URCA process, as found by Dall'Osso et al. (2009), then the inclination angle evolves following

$\frac{\sin ^{2} \chi}{1+3 \cos ^{2} \chi}=\frac{\sin ^{2} \chi_{i}}{1+3 \cos ^{2} \chi_{i}}\left(\frac{t}{30 \mathrm{~s}}+1\right)^{240 / C}$,

where $\chi_{i}$ is the initial inclination angle, which is usually small, and $C \sim 2.3 \times 10^{-4} B_{\mathrm{t}, 14}^{2} M_{1.4}^{-1} v_{3}^{-2}$. Thus the magnetic dipole moment will be orthogonal to the rotation axis in less than $\sim 1 \mathrm{~ms}$ when $\chi_{i}=1^{\circ}$. For the APR equation of state, we obtain $\tilde{I}=0.283$ for a neutron star with mass $1.4 M_{\odot}$ and radius $R=11.5 \mathrm{~km}$ (Owen et al. 1998). The timescale $t_{\mathrm{B}, \mathrm{gw}}$ with $\chi=\pi / 2$ reads (Cuofano et al. 2012)

$t_{\mathrm{B}, \mathrm{gw}} \approx 3.8 \times 10^{14} M_{1.4}^{-1} R_{11.5}^{-2} B_{\mathrm{t}, 14}^{-4} \mathrm{v}_{3}^{-4} \mathrm{~s}$.

The surface magnetic field also slows down the magnetar. For simplicity, the magnetic dipole radiation model is adopted. The torque due to magnetic dipole radiation and corresponding timescale are then given by

$N_{\text {dip }} \equiv-\frac{\mu^{2} \Omega^{3} \sin ^{2} \chi}{6 c^{3}}=-\frac{\mu^{2}}{6 R_{\mathrm{L}}^{3}}$,

and

$t_{\text {dip }} \equiv I \Omega / N_{\text {dip }}=-1.4 \times 10^{3} B_{15}^{-2} v_{3}^{-2} \mathrm{~s}$,

where $t_{\text {dip }}$ is the typical timescale due to magnetic dipole radiation and we set $\chi=\frac{\pi}{2}$. $R_{\mathrm{L}}$ is the radius of the light cylinder

$R_{\mathrm{L}}=\frac{c}{\Omega} \approx 48 \mathrm{v}_{3}^{-1} \mathrm{~km}$, where $c$ is the speed of light. The matter outside $R_{\mathrm{L}}$ no longer interacts with the magnetar.

\subsection{Hypercritical accretion of a magnetar}

Previous studies of the $r$-mode instability in accretion neutron stars have focussed on LMXB systems (Andersson et al. 1999; Brown \& Ushomirsky 2000; Yoshida \& Eriguchi 2000; Bondarescu et al. 2007). Here we consider hypercritical fallback accretion of newborn rapidly rotating magnetars, where the accretion torque can be either positive or negative. Following MacFadyen et al. (2001), Zhang et al. (2008) and Piro \& Thrane (2012), we parameterize the fall-back accretion rate as

$\dot{M}=\left(\dot{M}_{\text {early }}^{-1}+\dot{M}_{\text {late }}^{-1}\right)^{-1}$,

where

$\dot{M}_{\text {early }}=10^{-3} \eta t^{1 / 2} M_{\odot} \mathrm{s}^{-1}$

and

$\dot{M}_{\text {late }}=10^{-3} \eta t_{0}^{13 / 6} t^{-5 / 3} M_{\odot} \mathrm{s}^{-1}$,

where $\eta \approx 0.01-10$ is a factor which accounts for explosion energies, $t$ is in units of seconds and $t_{0} \sim 10^{2}-10^{3} \mathrm{~s}$ is the typical time at which the accretion rate starts to decrease (MacFadyen et al. 2001). At late times, the accretion rate decreases as $\dot{M} \propto t^{-5 / 3}$ (Chevalier 1989). We thus obtain the total baryonic mass of the neutron star at an arbitrary time $t$,

$M_{b}(t)=M_{b, i}+\int_{0}^{t} \dot{M}_{b} d t$,

where $M_{b, i}$ is the initial baryonic mass of the magnetar. A fraction of the accreted mass will turn into the binding energy that will be radiated away in the form of neutrinos. Hence, the gravitational mass $(M)$ is more applicable (Lattimer \& Prakash 2001),

$M=M_{b}(t)\left[1+\frac{3}{5} \frac{G M_{b}(t)}{R c^{2}}\right]^{-1}$.

We take $R$ to be a constant in this paper. In reality, the radius $R$ changes especially when the mass grows very large (Lattimer \& Prakash 2001). However, a small change of $R$ cannot affect our results significantly, since the timescales acting on the $r$-mode mainly depend on the core temperature and the spin.

In order to parameterize the torques due to accretion and magnetic dipole radiation, we introduce two useful radii. The first one is the co-rotation radius where the Keplerian angular velocity of the disk equals the angular velocity of the neutron $\operatorname{star} \Omega$,

$r_{\mathrm{c}}=\left(\frac{G M}{\Omega^{2}}\right)^{1 / 3} \approx 16.5 M_{1.4}^{1 / 3} \mathrm{v}_{3}^{-2 / 3} \mathrm{~km}$.

The second one is the magnetospheric (Alfvén) radius where the ram pressure of the fall-back materials balances the magnetic pressure,

$r_{\mathrm{m}}=\left(\frac{\mu^{4}}{G M \dot{M}_{b}^{2}}\right)^{1 / 7} \approx 31.2 B_{15}^{4 / 7} R_{11.5}^{12 / 7} M_{1.4}^{-1 / 7} \dot{M}_{-3}^{-2 / 7} \mathrm{~km}$,

where $\mu=B R^{3}$ is the magnetic dipole moment and $\dot{M}_{-3}=$ $\dot{M}_{b} /\left(10^{-3} M_{\odot} / \mathrm{s}\right)$.

Usually the fastness parameter $\omega$ is defined as the ratio of the stellar angular velocity and the Keplerian angular velocity at 
the magnetospheric radius $r_{\mathrm{m}}$ (we refer to e.g., Elsner \& Lamb 1977; Ghosh \& Lamb 1979),

$$
\begin{aligned}
\omega & =\frac{\Omega}{\sqrt{G M / r_{\mathrm{m}}^{3}}}=\left(\frac{r_{\mathrm{m}}}{r_{\mathrm{c}}}\right)^{3 / 2} \\
& \approx 2.6 B_{15}^{6 / 7} R_{11.5}^{18 / 7} M_{1.4}^{-5 / 7} \dot{M}_{-3}^{-3 / 7} v_{3} .
\end{aligned}
$$

Using these parameters, we can parameterize the accretion torque and corresponding timescale through

$$
N_{\mathrm{acc}} \equiv \dot{M}_{b} \sqrt{G M r_{\mathrm{m}}} n(\omega)=n(\omega) \mu^{2} / r_{\mathrm{m}}^{3},
$$

and

$$
t_{\mathrm{acc}} \equiv I \Omega / N_{\mathrm{acc}}=180 \mathrm{v}_{3} \dot{M}_{-3}^{-1}\left(r_{\mathrm{m}} / 18 \mathrm{~km}\right)^{1 / 2} n(\omega)^{-1} \mathrm{~s} .
$$

If $r_{\mathrm{m}}<r_{\mathrm{c}}$, the accreted matter transfers its angular momentum to the magnetar, and thus provides a positive torque. Otherwise, the fall-back matter acts as a propeller and offers a negative torque. A detailed picture of accretion onto magnetic stars has been elucidated by many authors, for example Pringle \& Rees (1972), Illarionov \& Sunvaev (1975), Ghosh \& Lamb (1979), Aly (1980), Eksi et al. (2005), and Dai \& Liu (2012). The expression of $n(\omega)$ differs slightly in different models. For simplification, we here adopt $n(\omega)=1-\omega$, as in Piro \& Ott (2011). This form of $n(\omega)$ includes the main properties of an interaction between the accretion disk and the magnetar. Furthermore, it has two advantages; $n(\omega)$ is continuous for all $\omega$ and becomes zero when $r_{\mathrm{m}}=r_{\mathrm{c}}$.

\subsection{Evolution of the $r$-mode}

We quantify the $r$-mode model based on Owen et al. (1998). The total angular momentum $J$ is given by

$J=I \Omega+J_{\mathrm{c}}$,

where $J_{\mathrm{c}}$ is the canonical angular momentum of the $r$-mode (Owen et al. 1998),

$J_{\mathrm{c}}=-\frac{3}{2} \alpha^{2} \tilde{J} M R^{2} \Omega$,

where $\tilde{J}=\int_{0}^{R} \rho r^{6} d r /\left(M R^{4}\right)$ and for a neutron star with the APR EOS, whose mass is $1.4 M_{\odot}$ and radius is $R=11.5 \mathrm{~km}$, we have $\tilde{J}=1.81 \times 10^{-2}$ (Owen et al. 1998). The canonical energy of the $r$-mode is given by (Owen et al. 1998),

$E_{\mathrm{c}}=\frac{1}{2} \alpha^{2} \tilde{J} M R^{2} \Omega^{2}$.

We obtain evolution of the $r$-mode using the time derivative of $J$ and $E_{\mathrm{c}}$ (Owen et al. 1998; Andersson et al. 2001; Cuofano et al. 2012),

$$
\begin{gathered}
\frac{d J}{d t}=\frac{3 \alpha^{2} \tilde{J} M R^{2} \Omega}{t_{\mathrm{gw}}}+N_{\mathrm{acc}}+N_{\mathrm{dip}}+N_{\mathrm{B}, \mathrm{gw}}, \\
\frac{d E_{\mathrm{c}}}{d t}=-2 E_{\mathrm{c}}\left(\frac{1}{t_{\mathrm{gw}}}+\frac{1}{t_{\mathrm{sv}}}+\frac{1}{t_{\mathrm{bv}}}+\frac{1}{t_{\mathrm{B}, \mathrm{t}}}\right),
\end{gathered}
$$

where the torques due to accretion $\left(N_{\mathrm{acc}}\right)$ and magnetic dipole radiation $\left(N_{\text {dip }}\right)$ affect the total stellar angular momentum together.

Substituting equations (22), (23), and (24) into equations (25) and (26), we obtain the evolution equations of $\Omega$ and $\alpha$,

$$
\begin{aligned}
\frac{1}{\Omega} \frac{d \Omega}{d t}=-\frac{\dot{M}_{b}}{A_{+} M} & +\frac{1}{A_{+}}\left(\frac{1}{t_{\mathrm{dip}}}+\frac{1}{t_{\mathrm{acc}}}+\frac{1}{t_{\mathrm{B}, \mathrm{gw}}}\right) \\
& -\frac{3 \alpha^{2} \tilde{J}}{\tilde{I} A_{+}}\left(\frac{1}{t_{\mathrm{sv}}}+\frac{1}{t_{\mathrm{bv}}}+\frac{1}{t_{\mathrm{B}, \mathrm{t}}}\right),
\end{aligned}
$$

and

$$
\begin{aligned}
\frac{1}{\alpha} \frac{d \alpha}{d t} & =\frac{\dot{M}_{b} A_{-}}{2 M A_{+}}-\frac{A_{-}}{A_{+}}\left(\frac{1}{t_{\mathrm{sv}}}+\frac{1}{t_{\mathrm{bv}}}+\frac{1}{t_{\mathrm{B}, \mathrm{t}}}\right) \\
& -\frac{1}{A_{+}}\left(\frac{1}{t_{\mathrm{dip}}}+\frac{1}{t_{\mathrm{acc}}}+\frac{1}{t_{\mathrm{B}, \mathrm{gw}}}\right)-\frac{1}{t_{\mathrm{gw}}},
\end{aligned}
$$

where $A_{ \pm}=1 \pm 3 \alpha^{2} \tilde{J} / 2 \tilde{I}$. We see that accretion and magnetic dipole radiation affect the amplitude and spin evolution simultaneously. The magnetic braking increases the $r$-mode amplitude while it spins down the magnetar. The effect of accretion is slightly complicated, since accretion can either spin up or down the magnetar while it can also either decrease or increase the $r$-mode amplitude.

In order to solve the above evolution equations, we must also know how the interior temperature of the magnetar evolves. The fall-back accretion will start $\sim 100$ s later after the stellar birth (MacFadyen et al. 2001; Dai \& Liu 2012). Meanwhile, the proto-neutron star will become a neutron star $\sim 50$ s later after its birth, and at this time the neutron star is transparent to neutrinos (Ferrari et al. 2003). Thus we consider a hot young isothermal neutron star with an initial temperature of $T_{0}=10^{10} \mathrm{~K}$. The newborn neutron star is then cooled down by the modifiedURCA processes, whose luminosity is $L_{v} \approx 3.5 \times 10^{39} T_{0}^{8} \mathrm{erg} / \mathrm{s}$ (Shapiro \& Teukolsky 1986; Alford \& Schwenzer 2014b). The direct URCA processes might set in when the star grows heavy enough. But since we only consider the time (the first $\sim 10^{4} \mathrm{~s}$ ) when gravitational radiation is significant, the direct URCA processes do not significantly affect our results. We also take into account the heating effect due to shear viscosity in this paper, namely, $H_{\mathrm{v}}=2 E_{\mathrm{c}} / t_{\mathrm{sv}}$. We do not consider the heating effect of accreted materials, since these materials have been cooled down by neutrinos in the accretion disk (Kohri \& Mineshige 2002; Zhang \& Dai 2010) and the accretion column (Piro \& Ott 2011). The temperature evolution is then given by

$\frac{d T}{d t}=\frac{H_{\mathrm{v}}-L_{v}}{C_{V}}$,

where $C_{V} \approx 7.0 \times 10^{38} T_{9}$ erg K ${ }^{-1}$ (Shapiro \& Teukolsky 1986) is the heat capacity of the neutron star.

\section{Results}

Integrating equations (6), 27), 28) and (29), we can obtain evolution of the $r$-mode amplitude, spin, and temperature. The initial spin is of the order of one millisecond, but since accretion spins up the star near the mass-shedding limit, the effect of the initial spin is less important than that of accretion. Therefore, we mainly focus on effects of accretion and initialize the spin at $P_{0}=1 / v_{0}=3 \mathrm{~ms}$. The initial baryonic mass and radius of the magnetar in our model are taken to be $M_{b, i}=1.4 M_{\odot}$ and $R=11.5 \mathrm{~km}$, and thus the gravitational mass is $M_{i} \simeq 1.26 M_{\odot}$. The initial amplitude of the $r$-mode is fixed at $10^{-8}$.

Fig. 1 gives four examples of evolution of the $r$-mode amplitude $(\alpha)$, spin $(v)$ and toroidal magnetic field $\left(B_{\mathrm{t}}\right)$. We study two specific accretion rates; $\eta=0.01$ with $t_{0}=3000 \mathrm{~s}$ (red lines) and $\eta=0.1$ with $t_{0}=300 \mathrm{~s}$ (blue lines). The magnetic field is typically chosen to be $B=2 \times 10^{14} \mathrm{G}$ (solid lines) and $B=5 \times 10^{14} \mathrm{G}$ (dashed lines). For comparison, we also study the case without accretion for $B=2 \times 10^{14} \mathrm{G}$ (black solid line). It is shown that the combination of accretion and magnetic fields can significantly affect the $r$-mode amplitude evolution. If the accretion can spin up the neutron star significantly, the amplitude 
grows quickly to the maximum value of $\sim 0.001$. Otherwise, the growth speed might be considerably suppressed. In the following, we use a semi-analytical method to analyze the amplitude and spin evolution.

To find which mechanism dominates the evolution, we compare the terms in the right hand side of equation (27) or (28). We take the pre-factor $A_{ \pm} \approx 1$, since the amplitude is very small in an early stage. Since the viscosity terms and the gravitational wave terms due to magnetic deformation are very small, we mainly consider the other terms. Firstly, we compare the accretion torque with the first term in equation 27), that is,

$\left|\frac{M}{\dot{M}_{b} t_{\mathrm{acc}}}\right| \approx 11 B_{15}^{2 / 7} R_{11.5}^{-8 / 7} M_{1.4}^{3 / 7} \dot{M}_{-3}^{-1 / 7} v_{3}^{-1}|n(\omega)|$.

Generally, $v<10^{3} \mathrm{~Hz}$ holds in most of the evolution time (Fig.11) and $n(\omega)$ is in the range of -0.5 to 0.5 , so we treat with the accretion torque as the dominant term over the first term.

The torque due to magnetic dipole radiation is smaller than the accretion torque, since the magnetospheric radius is generally much smaller than the light cylinder radius and $n(\omega) \in$ $[-0.5,0.5]$ in the amplitude growing stage so that

$\left|\frac{t_{\mathrm{dip}}}{t_{\mathrm{acc}}}\right|=\left|6\left(\frac{R_{\mathrm{L}}}{r_{\mathrm{m}}}\right)^{3} n(\omega)\right|>1$.

The toroidal magnetic field damping term is comparable to the accretion term, if the toroidal magnetic field grows strong enough, since we have

$\frac{t_{\mathrm{B}, \mathrm{t}}}{t_{\mathrm{acc}}} \approx 0.04 B_{\mathrm{t}, 14}^{-1} B_{15}^{-5 / 7} R_{11.5}^{-1 / 7} M_{1.4}^{13 / 14} \dot{M}_{-3}^{6 / 7} n(\omega)$.

However, for the spin evolution (equation 27), there is a prefactor $3 \alpha^{2} \tilde{J} / \tilde{I} A_{+}$. Considering in this paper (Fig. 1) that the amplitude is generally much smaller than 0.2 , we can assume that the accretion term dominates the spin evolution.

Adopting equations (13) and (14) and considering only the accretion term in the right-hand side of equation (27), we obtain an approximate spin evolution,

$v \approx\left\{\begin{array}{l}v_{0}+3.7 n(\omega) \eta t^{3 / 2}, \quad \text { if } t \lesssim t_{0}, \\ v_{c}+8.3 n(\omega) t_{0}^{3 / 2} \eta\left[1-\left(t_{0} / t\right)^{2 / 3}\right], \text { if } t \gtrsim t_{0},\end{array}\right.$

where $\quad v_{c}=v_{0}+3.7 n(\omega) \eta t_{0}^{3 / 2}$. Substituting equation (33) into equation (19), we find that $n(\omega)=0$ happens at $t=1.5 B_{15}^{4}\left(v_{0,333}\right)^{14 / 3} \eta^{-2} \mathrm{~s}$ or $t=$ $110 \eta^{3 / 5} B_{15}^{-6 / 5}\left(v_{c, 800}^{-7 / 5}\right)\left(t_{0,100}\right)^{13 / 10} \mathrm{~s}$, where $v_{0,333}=v_{0} / 333 \mathrm{~Hz}$, $\mathrm{v}_{c, 800}=\mathrm{v}_{c} / 800 \mathrm{~Hz}$ and $t_{0,100}=t_{0} / 100 \mathrm{~s}$. The second solution $\left(t \sim 110 \eta^{3 / 5}\left(t_{0,100}\right)^{13 / 10} \mathrm{~s}\right)$ corresponds to the time when the maximum spin is reached, which hints that before $\sim t_{0}$ the spin is increasing. This analytical expression for $v$ is consistent with the result shown in Fig.1.

The amplitude evolution begins to be affected by the gravitational wave emission when

$\frac{\left|-t_{\mathrm{gw}}\right|}{t_{\mathrm{acc}}} \approx 0.2 B_{15}^{2 / 7} R_{11.5}^{-36 / 7} M_{1.4}^{-11 / 7} \dot{M}_{-3}^{6 / 7} v_{3}^{-7} n(\omega)>1$.

This means that gravitational radiation dominates when $v>$ $794 B_{15}^{2 / 49} \dot{M}_{-3}^{6 / 49}|n(\omega)|^{1 / 7} \mathrm{~Hz}$. Approximately, it is required that $\mathrm{v}_{c}>794 B_{15}^{2 / 49} \dot{M}_{-3}^{6 / 49}|n(\omega)|^{1 / 7}$, which is roughly $3.7 n(\omega) \eta t_{0}^{3 / 2}>$ $794 B_{15}^{2 / 49} \eta^{6 / 49} t_{0}^{3 / 49}|n(\omega)|^{1 / 7}-v_{0}$.
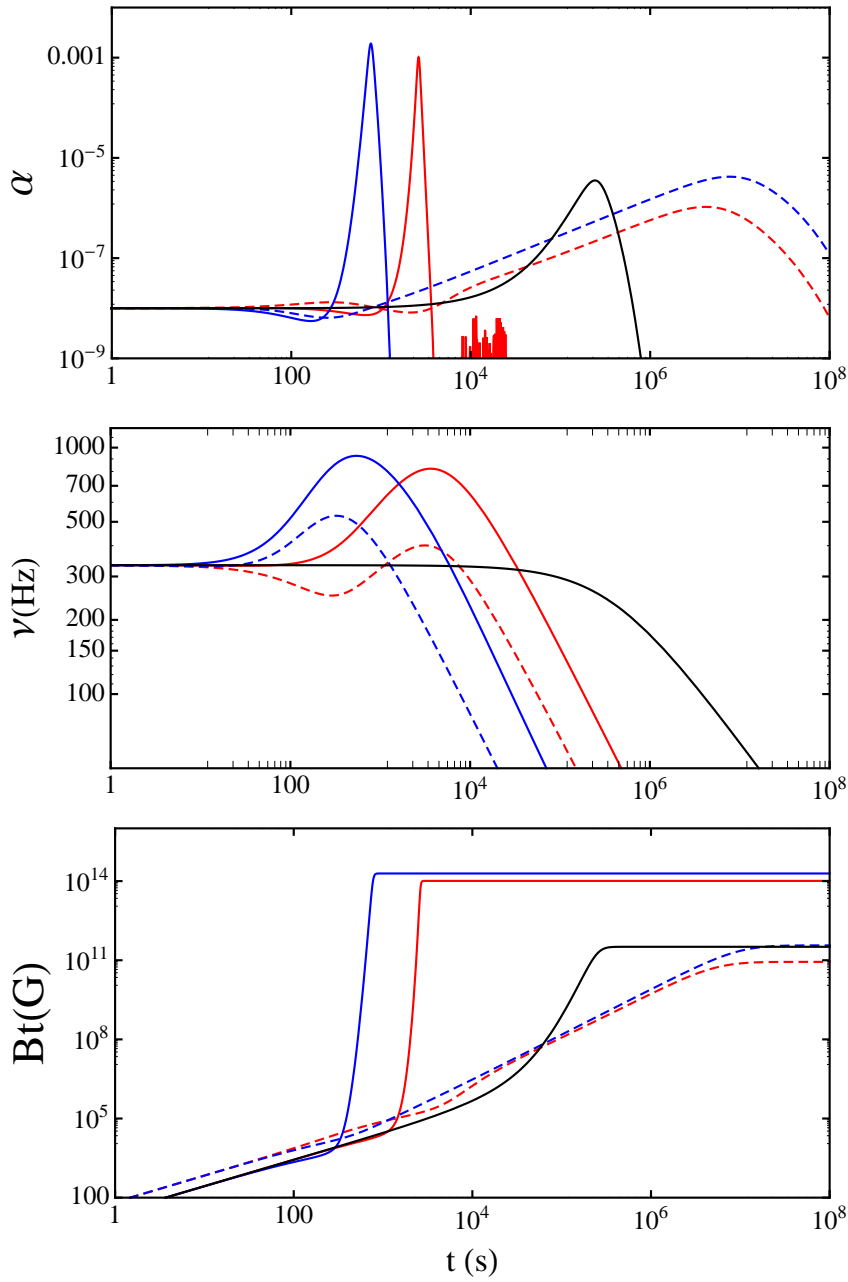

Fig. 1: The r-mode amplitude $(\alpha)$, spin $(v)$ and toroidal magnetic field $\left(B_{\mathrm{t}}\right)$ as functions of time. The black solid line is plotted for $B=2 \times 10^{14} \mathrm{G}$ and $\eta=0$. The red solid or dashed lines are obtained in the case of $\eta=0.01$ and $t_{0}=3000 \mathrm{~s}$ for $B=$ $2 \times 10^{14} \mathrm{G}$ or $B=5 \times 10^{14} \mathrm{G}$. The blue solid or dashed lines are obtained in the case of $\eta=0.1$ and $t_{0}=300 \mathrm{~s}$ for $B=2 \times 10^{14} \mathrm{G}$ or $B=5 \times 10^{14} \mathrm{G}$.

We also numerically show that the timescales $-t_{\mathrm{gw}}$ (purple lines), $t_{\mathrm{B}, \mathrm{t}}$ (brown lines) and $t_{\mathrm{acc}}$ (orange lines) evolve with time in Fig. 2. The accretion rate is fixed to be $\eta=0.01$ at $t_{0}=3000 \mathrm{~s}$. The solid lines correspond to the case of $B=2 \times 10^{14} \mathrm{G}$ and dashed lines to $B=5 \times 10^{14} \mathrm{G}$. The sharp transitions happen when $\omega \rightarrow 1$, which leads to $t_{\text {acc }} \rightarrow \infty$.

The amplitude is suppressed by the positive accretion torque before $\sim t_{0}$. After that, if the spin of the neutron star increases significantly enough, which leads to gravitational radiation dominating the $r$-mode amplitude $\left(\left|t_{\mathrm{gW}}\right|<\left|t_{\mathrm{acc}}\right|\right)$, rapid growth of the amplitude occurs (Fig.11). To calculate this rapid growth, we only consider the contribution of gravitational radiation in equation (28), and obtain

$\gamma \equiv \ln \left(\frac{\alpha}{\alpha_{0}}\right) \approx \frac{1}{21} \int_{0}^{t} v_{3}^{6} d t$ 

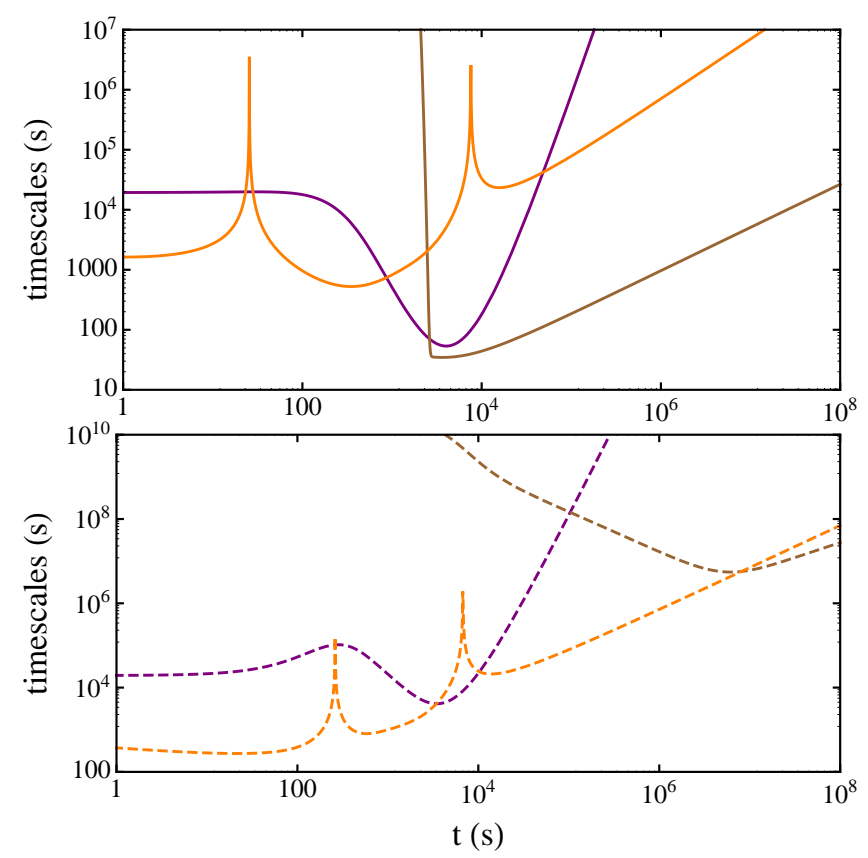

Fig. 2: The evolution of timescales $-t_{\mathrm{gw}}$ (purple lines), $t_{\mathrm{B}, \mathrm{t}}$ (brown lines) and $t_{\mathrm{acc}}$ (orange lines) in the cases with $\eta=0.01$ and $t_{0}=3000 \mathrm{~s}$. The solid lines correspond to the case of $B=$ $2 \times 10^{14} \mathrm{G}$ and dashed lines to $B=5 \times 10^{14} \mathrm{G}$.

Substituting equation (33) into this equation, we find

$\gamma \approx 4.7 \times 10^{-20} \int_{0}^{t}\left(v_{0}+3.7 n(\omega) \eta t^{3 / 2}\right)^{6} d t$.

The rapid growth happens only when $v_{0}<3.7|n(\omega)| \eta t^{3 / 2}$, in which case we can ignore the $v_{0}$ term in equation (36). Therefore, we get

$\gamma=12(n \eta)^{6}(t / 100 s)^{10}$,

which is strongly dependent on $n, \eta$ and $t$, meaning the amplitude can grow quickly with time. To reach saturation, the amplitude should grow up to $\alpha_{s} / \alpha_{0}=10^{3}-10^{8}$, which means $\gamma=\ln \left(\alpha_{s} / \alpha_{0}\right) \approx 7-18$. This implies that gravitational radiation can drive $r$-mode to saturation in a few hundred seconds if the star accretes a significant amount of matter. In this case, the maximum amplitude is reached when $t_{\mathrm{gw}}+t_{\mathrm{B}, \mathrm{t}} \approx 0$, which is $B_{\mathrm{t}, 14} \approx 0.24 R_{11.5}^{3} M_{1.4}^{2} B_{15}^{-1} \mathrm{v}_{3}^{7}$.

However, if the spin grows very slowly, the accretion torque will dominate the amplitude growth $\left(\left|t_{\mathrm{gw}} / t_{\mathrm{acc}}\right|>1\right)$. Considering only the accretion torque term in equation (28) and using equation (33), we approximately obtain the growth rate of the $r$-mode amplitude due to accretion,

$\gamma=\frac{8.3 n(\omega) t_{0}^{3 / 2} \eta\left[1-\left(t_{0} / t\right)^{2 / 3}\right]}{v_{c}+8.3 n(\omega) t_{0}^{3 / 2} \eta\left[1-\left(t_{0} / t\right)^{2 / 3}\right]}$,

where $n(\omega)$ is negative and $\alpha_{0}^{\prime}$ is the amplitude when the accretion torque becomes dominant. In this case, the amplitude grows very slowly with time. Then, the maximum amplitude is reached when $t_{\mathrm{B}, \mathrm{t}}+t_{\mathrm{acc}} \approx 0$, which is $B_{\mathrm{t}, 14}=$ $-0.04 B_{15}^{-5 / 7} R_{11.5}^{-1 / 7} M_{1.4}^{13 / 14} \dot{M}_{-3}^{6 / 7} n(\omega)$, where $n(\omega)<0$.

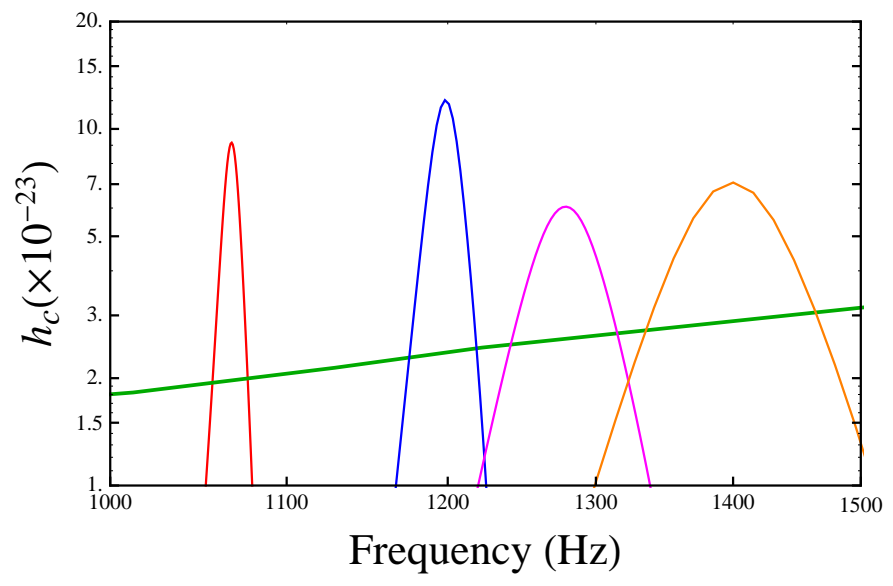

Fig. 3: The characteristic strain amplitude of the $r$-mode instability for accreting magnetars locating at a distance $D=1 \mathrm{Mpc}$. The green curve corresponds to ET noise rms strain amplitude. The red, blue, magenta, and orange curves correspond to the cases: $B=2 \times 10^{14} \mathrm{G}, \eta=0.01$, and $t_{0}=3000 \mathrm{~s} ; B=2 \times 10^{14} \mathrm{G}$, $\eta=0.1$, and $t_{0}=300 \mathrm{~s} ; B=5 \times 10^{14} \mathrm{G}, \eta=1$, and $t_{0}=100 \mathrm{~s}$; and $B=5 \times 10^{14} \mathrm{G}, \eta=10$, and $t_{0}=15 \mathrm{~s}$, respectively.

\section{Detectability of gravitational waves}

When the $r$-mode is unstable in a magnetar, gravitational waves are emitted. The $r$-mode became very interesting after gravitational waves from the merger of double black holes were detected by aLIGO (Abbott et al. 2016). We now test whether or not such a gravitational wave signal from a single source can be detectable by gravitational observatories such as ET. The gravitational wave strain amplitude for the $r$-mode, which is averaged over polarizations and orientations, is given by Owen et al. (1998) as

$h=\sqrt{\frac{3}{80 \pi}} \frac{\omega^{2} S_{22}}{D}$,

where $D$ is the distance of the source and

$S_{22}=\sqrt{2} \frac{32 \pi}{15} \frac{G M}{c^{5}} \alpha \Omega R^{3} \tilde{J}$,

where $c$ is the speed of light. We mainly focus on the frequency spectrum of the gravitational wave by using the Fouriertransform, $\tilde{h}(f)^{2}=h(t)^{2}\left|\frac{d t}{d f}\right|$ (Owen et al. 1998), where $f \approx$ $2 \Omega / 3 \pi$ is the frequency of the gravitational wave for the $l=m=$ 2 current multipoles. A characteristic amplitude $h_{c}=h \sqrt{f^{2}\left|\frac{d t}{d f}\right|}$ is usually defined and we show it in Fig. 3 for different cases versus the $r m s$ strain noise $h_{r m s}=\sqrt{f S_{h}(f)}$ of ET, where $S_{h}(f)$ is the noise strain amplitude.

The red, blue, magenta, and orange solid curves in Fig. 3 are the characteristic amplitude $h_{c}$ in the cases with different accretion rates and magnetic fields, when the sources locate at a distance of $D=1 \mathrm{Mpc}$. The green line is the ET noise $r m s$ strain amplitude (Hild et al.2011) and the characteristic amplitudes are higher than the noise curves. Thus it is possible to detect such sources once ET works.

\section{Discussion and Conclusion}

In this paper, we have explored the $r$-mode instability in newborn rapidly-rotating hyper-accreting magnetars. Their spin evo- 
lution is influenced by the accretion torque. There are two possibilities. Case I: If the neutron star is spun up significantly by accretion, namely, the parameters must satisfy the condition of $3.7 n(\omega) \eta t_{0}^{3 / 2}>794 B_{15}^{2 / 49} \eta^{6 / 49} t_{0}^{3 / 49}|n(\omega)|^{1 / 7}-v_{0}$, the $r$ mode amplitude will grow quickly due to the gravitational radiation $\ln \left[\alpha(t) / \alpha_{0}\right]=12(n \eta)^{6}(t / 100 \mathrm{~s})^{10}$. The saturation happens when the damping due to the generation of a toroidal magnetic field balances the gravitational radiation for $B_{\mathrm{t}, 14} \approx$ $0.24 R_{11.5}^{3} M_{1.4}^{2} B_{15}^{-1} v_{3}^{7}$. The generated magnetic field reaches a value that is slightly weaker than the poloidal field. The saturation amplitude is $\sim 0.001$.

However, if the spin-up is not significant (Case II), the accretion torque will dominate the amplitude growth, which leads to a much slower growth (equation 38). The saturation is then met when $B_{\mathrm{t}, 14}=0.04 B_{15}^{-5 / 7} R_{11.5}^{-1 / 7} M_{1.4}^{13 / 14} \dot{M}_{-3}^{6 / 7}|n(\omega)|$. The maximum saturation amplitude is of the order of $\sim 10^{-6}$ to $10^{-5}$. The generated toroidal field is much weaker than that in the case of a significant spin-up.

If the magnetar is sufficiently spun-up (i.e., Case I discussed here), the gravitational radiation from such a magnetar would be possibly detectable with the future mission ET, if the source were located at a distance $<1 \mathrm{Mpc}$. Core-collapse SNe and gammaray bursts are electromagnetic counterparts of such gravitational wave sources. However, based on the recent estimates (Li et al. 2011; Strolger et al. 2015), the local core-collapse supernova rate is $\sim$ afew $\times 10^{-2} \mathrm{Mpc}^{-3}$ per century. Thus, the detectable gravitational wave event rate with ET is as low as roughly one per millennium.

\section{Acknowledgements}

We thank the referee for valuable comments and constructive suggestions that have allowed us to improve our manuscript significantly. We also thank Yi-Ming Hu, Yan Yan and Yun-Wei Yu for their helpful discussions. This work is supported by the National Basic Research Program ("973" Program) of China (grant No. 2014CB845800) and the National Natural Science Foundation of China (grant No. 11573014).

\section{References}

Abbassi S., Rieutord M., Rezania V., 2012, MNRAS, 419, 2893

Abbott, B. P. et al. 2016, Phys. Rev. Lett., 116, 061102

Akmal A., Pandharipande V. R., Ravenhall D. G., 1998, PhRvC, 58, 1804

Alford M. G., Mahmoodifar S., Schwenzer K., 2012a, PhRvD, 85, 024007

Alford M. G., Mahmoodifar S., Schwenzer K., 2012b, PhRvD, 85, 044051

Alford M. G., Schwenzer K., 2014a, PhRvL, 113, 251102

Alford M. G., Schwenzer K., 2014b, ApJ, 781, 26

Alford M. G., Schwenzer K., 2015, MNRAS, 446, 3631

Aly J. J., 1980, A\&A, 86, 192

Andersson N., Jones D. I., Kokkotas K. D., 2002, MNRAS, 337, 1224

Andersson N., 1998, ApJ, 502, 708

Andersson N., Jones D. I., Kokkotas K. D., Stergioulas N., 2000, ApJ, 534, L75

Andersson N., Kokkotas K., Schutz B. F., 1999, ApJ, 510, 846

Andersson N., Kokkotas K. D., 2001, IJMPD, 10, 381

Andersson N., Kokkotas K. D., Stergioulas N., 1999, ApJ, 516, 307

Arras P., Flanagan E. E., Morsink S. M., Schenk A. K., Teukolsky S. A., Wasserman I., 2003, ApJ, 591, 1129

Arzoumanian Z., Chernoff D. F., Cordes J. M., 2002, ApJ, 568, 289

Asai H., Lee U., Yoshida S., 2015, MNRAS, 449, 3620

Bildsten L., Ushomirsky G., 2000, ApJ, 529, L33

Blackman E. G., Perna R., 2004, ApJ, 601, L71

Bondarescu R., Teukolsky S. A., Wasserman I., 2009, PhRvD, 79, 104003

Bondarescu R., Teukolsky S. A., Wasserman I., 2007, PhRvD, 76, 064019

Brown E. F., Ushomirsky G., 2000, ApJ, 536, 915

Chandrasekhar S., 1970, PhRvL, 24, 611

Chevalier R. A., 1989, ApJ, 346, 847
Chugunov A. I., 2015, MNRAS, 451, 2772

Corsi A., Mészáros P., 2009, ApJ, 702, 1171

Cutler C., 2002, PhRvD, 66, 084025

Cuofano C., Drago A., 2010, PhRvD, 82, 084027

Cuofano C., Dall'Osso S., Drago A., Stella L., 2012, PhRvD, 86, 044004

Dall'Osso S., Shore S. N., Stella L., 2009, MNRAS, 398, 1869

Dall'Osso S., Stratta G., Guetta D., Covino S., De Cesare G., Stella L., 2011, A\&A, 526, A121

Dall'Osso S., Giacomazzo B., Perna R., Stella L., 2015, ApJ, 798, 25

Dai Z. G., Liu R.-Y., 2012, ApJ, 759, 58

Dai Z. G., Lu T., 1998a, PhRvL, 81, 4301

Dai Z. G., Lu T., 1998b, A\&A, 333, L87

Dai Z. G., Wang S. Q., Wang J. S., Wang L. J., Yu Y. W., 2016, ApJ, 817, 132

Ekşi K. Y., Hernquist L., Narayan R., 2005, ApJ, 623, L41

Elsner R. F., Lamb F. K., 1977, ApJ, 215, 897

Ferrari V., Miniutti G., Pons J. A., 2003, MNRAS, 342, 629

Friedman J. L., Schutz B. F., 1978, ApJ, 222, 281

Friedman J. L., Morsink S. M., 1998, ApJ, 502, 714

Ghosh P., Lamb F. K., 1979, ApJ, 234, 296

Gusakov M. E., Chugunov A. I., Kantor E. M., 2014, PhRvD, 90, 063001

Harry G. M., LIGO Scientific Collaboration, 2010, CQGra, 27, 084006

Haskell B., 2015, IJMPE, 24, 1541007

Haskell B., Degenaar N., Ho W. C. G., 2012, MNRAS, 424, 93

Ho W. C. G., Andersson N., Haskell B., 2011, PhRvL, 107, 101101

Hild S., et al., 2011, CQGra, 28, 094013

Ho W. C. G., Lai D., 2000, ApJ, 543, 386

Illarionov A. F., Sunyaev R. A., 1975, A\&A, 39, 185

Kasen D., Bildsten L., 2010, ApJ, 717, 245

Kohri K., Mineshige S., 2002, ApJ, 577, 311

Kokkotas K. D., Stergioulas N., 1999, A\&A, 341, 110

Kouveliotou C., Dieters S., Strohmayer T., et al., 1998, Nature, 393, 235

Lattimer J. M., Prakash M., 2001, ApJ, 550, 426

Lee U., 2005, MNRAS, 357, 97

Levin Y., 1999, ApJ, 517, 328

Li W., Chornock R., Leaman J., Filippenko A. V., Poznanski D., Wang X., Ganeshalingam M., Mannucci F., 2011, MNRAS, 412, 1473

Lindblom L., Mendell G., 2000, PhRvD, 61, 104003

Lindblom L., Mendell G., Owen B. J., 1999, PhRvD, 60, 064006

Lindblom L., Owen B. J., Morsink S. M., 1998, PhRvL, 80, 4843

Lindblom L., Owen B. J., Ushomirsky G., 2000, PhRvD, 62, 084030

Lindblom L., Tohline J. E., Vallisneri M., 2001, PhRvL, 86, 1152

MacFadyen A. I., Woosley S. E., Heger A., 2001, ApJ, 550, 410

Madsen J., 2000, PhRvL, 85, 10

Madsen J., 1998, PhRvL, 81, 3311

Mahmoodifar S., Strohmayer T., 2013, ApJ, 773, 140

Manchester R. N., Hobbs G. B., Teoh A., Hobbs M., 2005, AJ, 129, 1993

Metzger B. D., Thompson T. A., Quataert E., 2007, ApJ, 659, 561

Michel F. C., 1988, Nature, 333, 644

Mytidis A., Coughlin M., Whiting B., 2015, ApJ, 810, 27

Owen B. J., Lindblom L., Cutler C., Schutz B. F., Vecchio A., Andersson N., 1998, PhRvD, 58, 084020

Papazoglou M. C., Moustakidis C. C., 2016, Ap\&SS, 361, 98

Piro A. L., Ott C. D., 2011, ApJ, 736, 108

Piro A. L., Thrane E., 2012, ApJ, 761, 63

Pringle J. E., Rees M. J., 1972, A\&A, 21,

Rezzolla L., Lamb F. K., Marković D., Shapiro S. L., 2001a, PhRvD, 64, 104013 Rezzolla L., Lamb F. K., Marković D., Shapiro S. L., 2001b, PhRvD, 64, 104014 Rezzolla L., Lamb F. K., Shapiro S. L., 2000, ApJ, 531, L139

Shapiro, S. L., \& Teuklosky, S. A., 1986, Black Holes, White Dwarfs, and Neutron Stars (New York: Wiley)

Shternin P. S., Yakovlev D. G., 2008, PhRvD, 78, 063006

Shternin P. S., Baldo M., Haensel P., 2013, PhRvC, 88, 065803

Strolger, L. G. et al., 2015, ApJ, 813, 93

Thompson C., Duncan R. C., 1996, ApJ, 473, 322

Thompson C., Duncan R. C., 1995, MNRAS, 275, 255

Thompson T. A., Chang P., Quataert E., 2004, ApJ, 611, 380

Usov V. V., 1992, Nature, 357, 472

Vasisht G., Gotthelf E. V., 1997, ApJ, 486, L129

Wang S. Q., Wang L. J., Dai Z. G., Wu X. F., 2015, ApJ, 799, 107

Woosley S. E., 2010, ApJ, 719, L204

Yang S.-H., Zheng X.-P., Pi C.-M., Yu Y.-W., 2010, MNRAS, 403, 2007

Yoshida S., Eriguchi Y., 2000, MNRAS, 316, 917

Yu Y.-W., Cao X.-F., Zheng X.-P., 2009, ApJ, 706, L221

Zhang D., Dai Z. G., 2010, ApJ, 718, 841

Zhang B., Mészáros P., 2001, ApJ, 552, L35

Zhang W., Woosley S. E., Heger A., 2008, ApJ, 679, 639

Zheng X.-P., Yu Y.-W., Li J.-R., 2006, MNRAS, 369, 376 